\title{
Do climate simulations from models forced by averaged sea surface temperatures represent actual dynamics?
}

\author{
P. J. Roebber ${ }^{1}$, A. A. Tsonis ${ }^{1}$ and J. B. Elsner ${ }^{2}$ \\ ${ }^{1}$ Department of Geosciences, University of Wisconsin at Milwaukee, 3209 N. Maryland, Milwaukee, WI, USA 53211 \\ ${ }^{2}$ Department of Meteorology. The Florida State University, Tallahassee, Florida, USA 32306 - 3034
}

Received 23 January 1997 - Accepted 2 September 1997

\begin{abstract}
Recently atmospheric general circulation models (AGCMs) forced by observed sea surface temperatures (SSTs) have offered the possibility of studying climate variability over periods ranging from years to decades. Such models represent an alternative to fully coupled asynchronous atmosphere ocean models whose long term integration remains problematic. Here, the degree of the approximation represented by this approach is investigated from a conceptual point of view by comparing the dynamical properties of a low order coupled atmosphereocean model to those of the atmospheric component of the same model when forced with monthly values of SST derived from the fully coupled simulation. The low order modeling approach is undertaken with the expectation that it may reveal general principles concerning the dynamical behavior of the forced versus coupled systems; it is not expected that such an approach will determine the details of these differences, for which higher order modeling studies will be required. We discover that even though attractor (global) averages may be similar, local dynamics and the resultant variability and predictability characteristics differ substantially. These results suggest that conclusions concerning regional climatic variability (in time as well as space) drawn from forced modeling approaches may be contaminated by an inherently unquantifiable error. It is therefore recommended that this possibility be carefully investigated using state-of-the-art coupled AGCMs.
\end{abstract}

\section{Introduction}

Because of the tremendous opportunity to enhance the predictive capability of AGCMs on seasonal (and longer) time scales, recent research has attempted to exploit the relationship between tropical SSTs and midlatitude climatic patterns (Kumar et al. 1994; Kumar and Hoerling 1995; Livezey et al. 1996) for the purpose of understanding climate variability and prediction. These models, which are forced by observed SSTs, represent an alternative to fully coupled asynchronous atmosphere ocean models (whose long term integration remains problematic). The details of the observed SST forcing varies, but essentially amounts to a periodic (e.g. Kumar and Hoerling 1995 use monthly values) updating of the AGCM boundary conditions represented by averaged SSTs. Livezey et al. (1996) recently evaluated this approach by contrasting the performance of AGCM forecasts whose updated boundary conditions were obtained from observed and forecast average SSTs (determined from a coupled general circulation model).

In this paper, the viability of this approximation (defined as the capability of continuing effectiveness) is investigated by posing a fundamental conceptual question: by separating the atmospheric and oceanic components of the coupled tropical-extratropical climate system, does one alter the dynamics of those systems in meaningful ways? The answer to this question is non-trivial, since from a non-linear dynamics point of view splitting the physical system into individual components alters the system by definition, even when the feedbacks are relatively weak. The salient question is whether the altered system behaves in a manner that is consistent with the objectives of making predictions on seasonal and longer time scales. One notable behavior of such complex systems is the possibility of obtaining spectacular results for individual cases without any assurance of continuing effectiveness: consistency in future performance depends crucially on the structure of the attractor that describes the system, since one unavoidably changes the intrinsic dynamics of the system by forcing the atmosphere with averaged SSTs. Since the answers to such questions can only be found through experimentation, the dynamics of fully coupled and forced low-order atmosphereocean models, introduced in section 2 , are explored (section 
Roebber et al.: Do climate simulations represent actual dynamics?

3). The implications of our results are discussed in section 4.

$$
\frac{d Z}{d t}=b X Y+X Z-Z
$$

\section{Atmosphere-Ocean Model}

In this paper, we resort to simulations with a low-order AGCM (Lorenz 1984), representing the evolution of the midlatitude atmosphere, which we couple with a simple delay oscillator model of the El Niño/Southern Oscillation (ENSO) phenomenon (Tziperman et al. 1994). The principal motivation for using a simple model rather than a fully coupled atmosphere-ocean model in this context is the enhanced opportunity to explore the coupled system dynamics while still retaining quantitative rigor. We note that the use of such simple models to gain valuable insights into complex problems has a rich history in meteorological research and is not preempted by the availability of more complex models. Lorenz $(1963 ; 1984 ; 1990)$ pioneered the use of such models in his efforts to explore the basic character of atmospheric predictability. Recently, Paimer (1993) used such an approach to examine the physical basis for extended range atmospheric predictions. Such models have also been widely used for conceptual studies of the general behavior of the deep ocean circulation (see Weaver and Hughes 1992 for a review). Statistical-dynamical models including energy balance models and radiative convective models have helped to elucidate basic features of the climate system (see MacCracken and Ghan 1987 for a review). We view the use of such models as complementary to more complex modeling efforts: accordingly, simple models might best be considered as tools to be used to help identify whether more complex modeling efforts are warranted.

In this study, the coupling of the Lorenz (1984) model of the atmospheric general circulation with the Tziperman et al. (1994) model of ENSO is motivated by the evidence of a subtle midlatitude atmospheric response to anomalous tropical SSTs associated with ENSO (Ropelewski and Halpert 1987; Livezey and Mo 1987; Trenberth et al. 1988; Glantz et al. 1991). While a basic pattern of response has been identified by researchers, the subtlety alluded to here is with respect to the magnitude and consistency of the midlatitude response to a particular tropical SST anomaly (see the concluding discussion for an example). The AGCM used herein is defined by three ordinary differential equations (Lorenz 1984):

$$
\begin{aligned}
& \frac{d X}{d t}=-Y^{2}-Z^{2}-a X+a F \\
& \frac{d Y}{d t}=X Y-b X Z-Y+G
\end{aligned}
$$

wherc $\mathrm{t}$ represents time, $\mathrm{X}, \mathrm{Y}$ and $\mathrm{Z}$ represent the meridional temperature gradient (or equivalently the strength of the zonal flow), and the amplitudes of the cosine and sine phases of a chain of superposed large scale eddies, respectively. These equations can be derived from the mean and perturbation quasi-geostrophic potential vorticity equations (see Holton, 1992, p.164-165), using a truncated Fourier series expansion of the geostrophic streamfunction. In eq. (1), F represents the meridional gradient of diabatic heating, and is the value to which $\mathrm{X}$ would be driven (over a damping time equal to $\mathrm{a}^{-1}$ ) in the absence of coupling between the westerly current and the eddies. The term $G$ in eq. (2) is the asymmetric thermal forcing, representing the longitudinal heating contrast between land and sea, and is the value to which Y would be driven (over a damping time of unity) in the absence of coupling. This coupling, which results in amplification of the eddies at the expense of the westerly current, is represented by the quadratic terms $X Y$ in eq. (2), $X Z$ in eq. (3) and $-Y^{2}-Z^{2}$ in eq. (1). Finally, the quadratic terms proportional to $b$ in (2) and (3) represent displacement of the eddies by the westerly current. Thus, for $a<1$ and $b>1$, the eddies damp more rapidly than the westerly flow and are displaced more rapidly than they amplify. The model exhibits a wide range of plausible atmospheric dynamical behaviors for particular values of F and G (Lorenz 1984, 1990; Masoller et al. 1992; Pielke and Zeng 1994; Rocbber 1995).

Ocean thermocline depth deviations from seasonal values at the eastern boundary of the equatorial Pacific are simulated by an heuristic model of the ENSO delay oscillator mechanism (Tziperman et al. 1994):

$$
\begin{aligned}
\frac{d h}{d t} & =\tau_{1} A\left(h\left\{t-\left[L /\left(2 C_{k}\right)\right]\right\}\right) \\
& -\tau_{2} A\left(h\left\{t-\left[L / C_{k}+L /\left(2 C_{R}\right)\right]\right\}\right) \\
& +\tau_{3} \cos \omega(t-\delta)
\end{aligned}
$$

where $t$ is time, $L$ is the basin width, $\omega$ is the annual frequency and the piece-wise function $A(h)$, defined in Munnich et al. (1991), relates wind stress to thermocline depth. The three terms on the right represent the wind-forced eastward traveling Kelvin wave of speed $C_{k}$, the westward traveling Rossby wave of speed $C_{R}$ and the equatorial seasonal forcing (representing variations in wind amplitude and SST), respectively. This simple model exhibits behavior that is qualitatively consistent with the behavior of ENSO as deduced from observational studies and more complex 


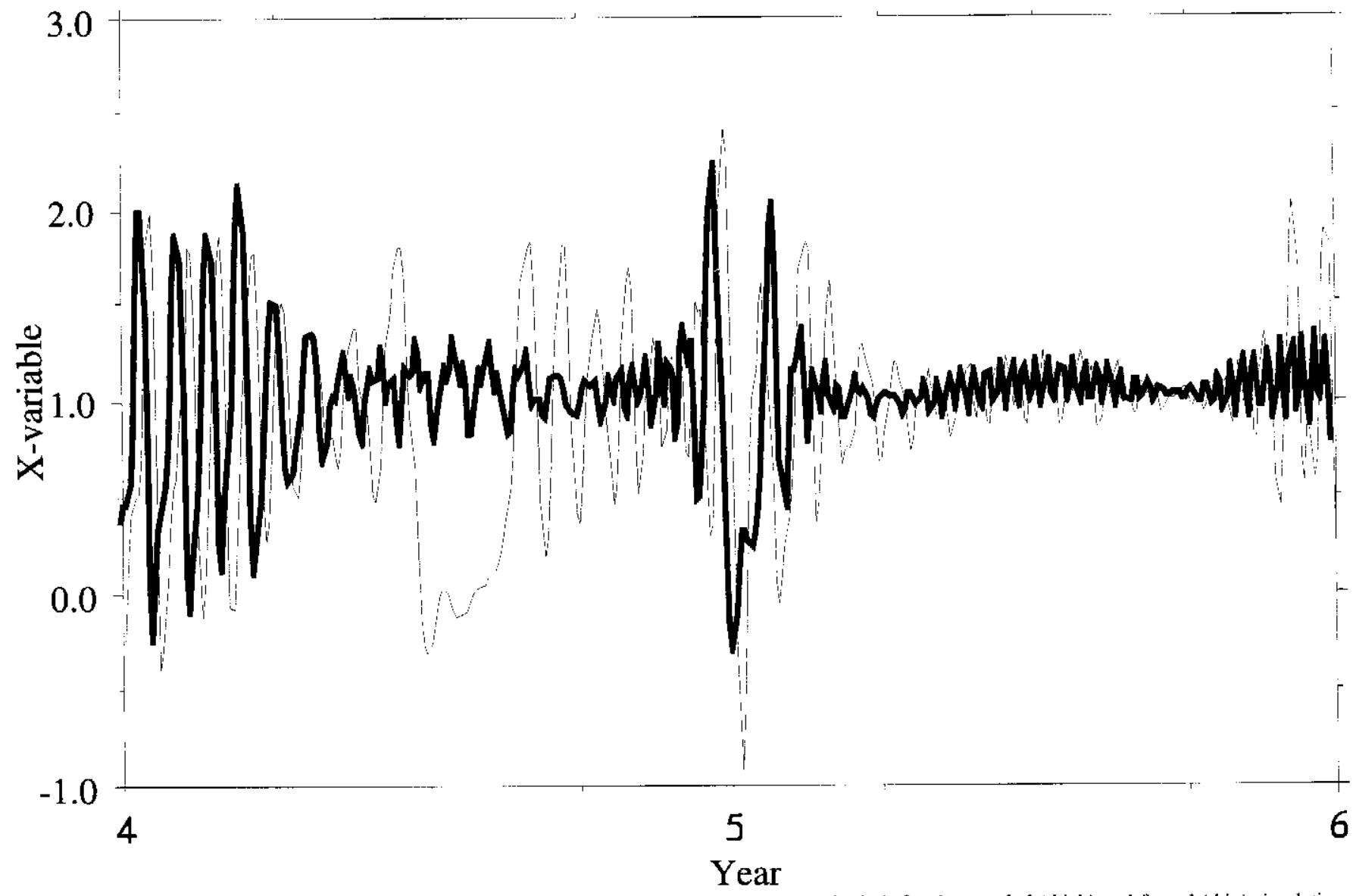

Fig. 1. Time series of the atmospheric model variable $X$ (representing the strength of the zonal wind) for the coupled (thick) and forced (thin) simulations during the transition from a warm to a cold ENSO phase in the years 4 to 6.

modeling efforts (e.g. Zebiak and Cane 1987).

At this point we have described an atmospheric model which simulates the dynamical behavior of the midlatitude atmospheric general circulation and a model of ENSO which reflects the essential interactions between the atmosphere and ocean in the equatorial Pacific. These models as they stand do not simulate the global consequences of ENSO, that is the midlatitude atmospheric response to SST anomalies in the equatorial Pacific (teleconnections). Since a primary means for interaction between equatorial SST anomalies and the midlatitude circulation must arise through adjustments in the meridional gradient in diabatic heating (e.g. Mechoso et al. 1987), we can simulate the global response to ENSO through adjustments to $F$ in eq. (1). Thus, we allow $F$ and $G$ to vary in a midlatitude seasonal cycle as in Roebber (1995) and we represent the midlatitude atmosphere-ocean coupling by superimposing upon $\mathrm{F}$ variations associated with tropical SST anomalies:

$$
\begin{aligned}
& F(t)=F_{0}+F_{1} \cos \omega t+F_{2} S S T \\
& G(t)=G_{0}+G_{1} \cos \omega t
\end{aligned}
$$

where SST' represents the tropical SST anomaly. These anomalies are obtained from thermocline depth deviations, $h$, by assuming that vertical displacements of isotherms are the dominant cause of the SST variations, with negligible time lags:

$$
S S T^{\prime}=T_{ \pm} \tanh \left(c_{ \pm} h\right)
$$

where the parameters $T_{+}$and $c_{+}\left(T_{-}\right.$and $\left.c_{-}\right)$are associated with positive (negative) thermocline depth deviations, $h$, obtained from (4). Thus, the coupled system represented by eq. (1)-(7) simulates the complex dynamics of the midlatitude atmospheric general circulation, which evolves through interaction between atmospheric eddies and the zonal current but is fundamentally driven by zonal and meridional gradients in diabatic heating, the latter being modified by the dynamics of ENSO (which is considered to evolve independently of midlatitude atmospheric adjustments).

\section{Coupled and Forced Simulations}

Equations 1-4 were integrated for 1024 years to remove all 

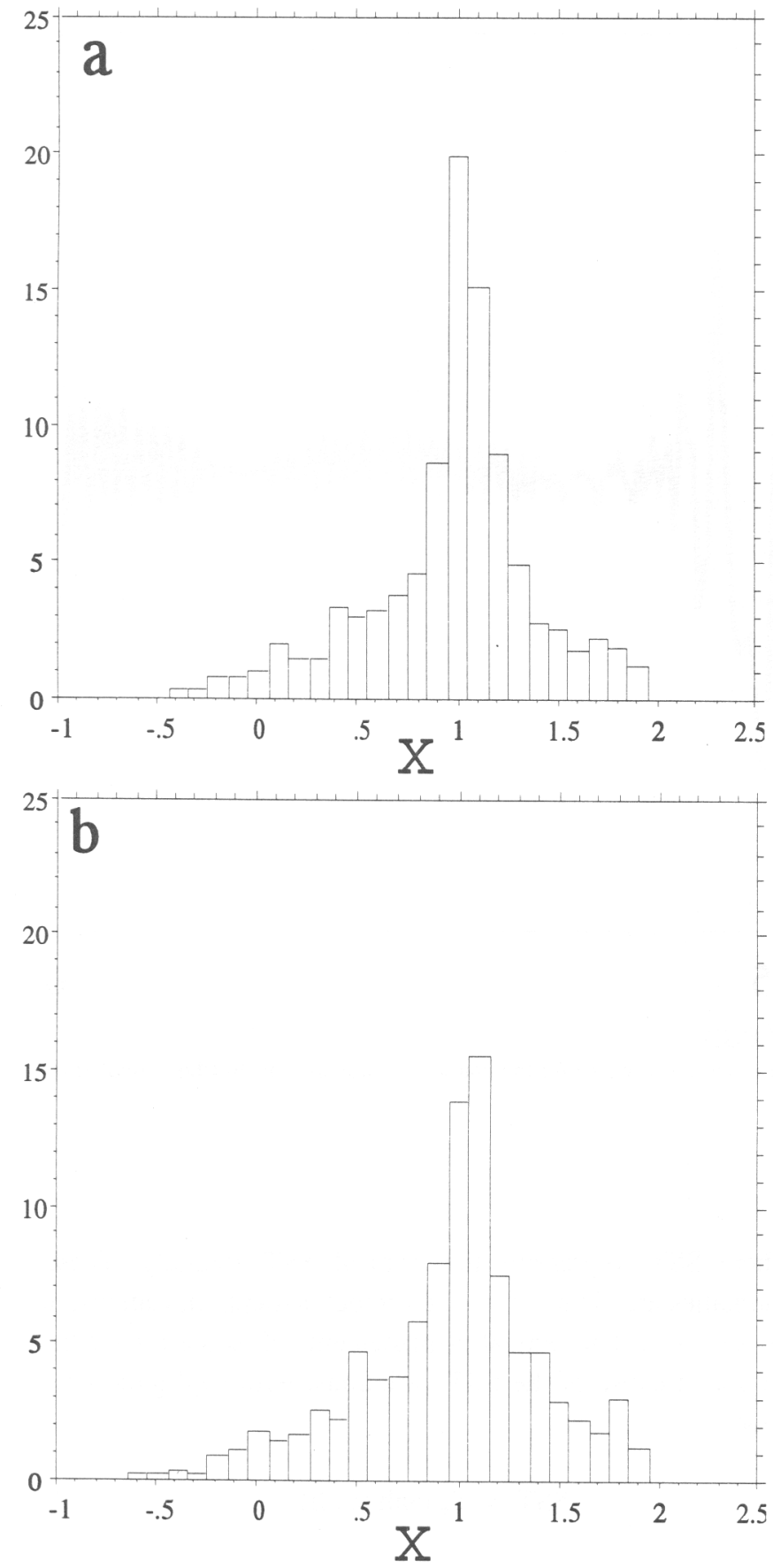

Fig. 2. The observed frequency distributions (percent) of the atmospheric variable $\mathrm{X}$ for the (a) coupled and (b) forced simulations for the 10 year period (every 30th point).

transients and then for an additional period for analysis purposes. The basic model parameters take the values used in Lorenz (1984; 1990) and Roebber (1995) for the atmospheric model and Tziperman et al. (1994) for ENSO. The value for $F_{2}$, which represents the adjustment in $F$ associated with equatorial SST anomalies (and thus the strength of the coupling between midlatitude dynamics and equatorial SSTs), was selected to be consistent with adjustments in meridional heating derived from AGCM simulations of the atmospheric response to ENSO (Mechoso et al. 1987). The values used in the simulations are: $\mathrm{a}=0.25$, $\mathrm{b}=4, \mathrm{C}_{\mathrm{k}}=\mathrm{L} /(2.3$ months $), \mathrm{C}_{\mathrm{R}}=\mathrm{C}_{\mathrm{k}} / 3, \mathrm{t}_{1}=(180 \text { days })^{-1}, \mathrm{t}_{2}=(120$ days $)^{-1}, \quad t_{3}=(138 \text { days })^{-1}, \quad \delta=6 \quad$ months (with $t=0$ corresponding to winter solstice), $\mathrm{F}_{0}=7, \mathrm{~F}_{1}=2, \mathrm{~F}_{2}=.5, \mathrm{G}_{0}=$ $.08, \mathrm{G}_{1}=-1.24, \mathrm{~T}_{+}=11, \mathrm{~T}_{-}=2, \mathrm{c}_{+}=0.8$ and $\mathrm{c}_{-}=1.9 . \mathrm{A}(\mathrm{h})$ is as in Munnich et al. (1991) with $a_{+}=a_{-}=1, b_{+}=1.5, b_{-}$ $=\mathrm{b}_{+} / 5, \mathrm{\kappa}=2.0$.

The integration of equations 1-4 with SST' obtained from (4) and (7) will be referred to as the coupled simulation, socalled because of the coupling of SST' with F and thus with $\mathrm{X}, \mathrm{Y}$ and $\mathrm{Z}$. It should be noted that this coupling is oneway, since adjustments in $\mathrm{X}, \mathrm{Y}$ and $\mathrm{Z}$ do not in turn feed back on SST' in the model (equatorial atmospheric forcing in the model is specified through the third term on the righthand side of (4)). In order to simulate the forcing of an AGCM with observed (averaged) SSTs, equations 1-4 were then integrated again for the same time period from the identical initial conditions, but the SST' used in (5) was obtained from monthly averages of the SST anomalies output from the (previously run) coupled simulation. This integration will be referred to as the forced simulation. The perturbations in SST, defined as the SST anomaly at a fixed time minus the monthly mean SST anomaly are in fact very small (less than a few tenths ${ }^{\circ} \mathrm{C}$ ). Thus, the sub-monthly variability in the model is comparable in magnitude to that of the real world. In contrast, the interannual variability is forced in the model by the ENSO phase, and is typically of the order of a few ${ }^{\circ} \mathrm{C}$ as observed. Despite the relatively small magnitude, one can not rule out the importance of the effect a priori, since we are altering the intrinsic dynamics of a complex nonlinear system with rich internal dynamics.

During the 10 year period of study in this paper, there were 3 ENSO events (with the warm phases peaking in the 1 st, 4th and 8th years) and the warm and cold phases tended to be associated with particular midlatitude climatic regimes. In the coupled simulation, the warm ENSO phases were often associated with extended winter seasons and in summer, either active (high amplitude fluctuations in the zonal jet) or blocking (weak or reversed meridional temperature gradient) regimes, while the cold phases resulted in reduced winter seasons and enhanced winter blocking. The forced simulation shows increased frequency of summertime blocking in place of active regimes. In addition, regimes identified by weak fluctuations in the strength of the westerlies, which frequently mark the transition from winter to summer in the coupled simulation, are less common in the forced mode.

Figure 1 shows the atmospheric circulations for the coupled and forced simulations during the transition from a warm ENSO phase to a cold phase in the years 4 to 6 (the full time series displays similar behavior but has been omitted for the sake of clarity). The coupled simulation 
exhibits a characteristic transition from an active warm ENSO winter in year 4 to an inactive summer and shortened, blocking winter during the cool down phase in year 5. In contrast, the forced simulation misses the seasonal transition and enters a summer blocking mode, finally readjusting to an approximately correct mode the following winter. The intermittent coherence in the two simulations is noteworthy: apparently the small errors introduced into the atmospheric forcing through monthly averaging of the SSTs are sufficient to occasionally lead the forced model atmosphere into different regions of the attractor, representing different climatic regimes. It is important to understand that this result represents a manifestation of the nonlinear dynamics of the system: the response is sometimes disproportionate to the forcing. One interpretation of this result is that by averaging SST, we change the fine scale details of the attractor which describes the dynamics of the coupled system, even though their gross structures remain similar. Thus, trajectories in phase space in the two systems follow slightly different paths. Most of the time, these differences are insignificant, but at particular times in their history, one trajectory may cross a critical point where a bifurcation can occur, leading to a quite different climatic response. In such instances, cause-effect relationships are unclear. We note that this type of behavior was qualitatively reproduced for a range of atmosphere-ocean coupling magnitudes represented by the parameter $\mathrm{F}_{2}$ (not shown). It is arguable that the forced model response depicted in Fig. 1 represents anomalous behavior resulting from the relatively large internal variability of the midlatitude atmosphere. To investigate this issue, we have rerun the forced simulation an additional 10 times, slightly modifying the initial conditions (by adding a random error of up to \pm 0.1 units to $\mathrm{X}, \mathrm{Y}$ and $\mathrm{Z}$, representing an error comparable to those quoted in the literature for temperature and winds from standard radiosonde measurements). This procedure naturally resulted in a range of forced model solutions, but the behavior of the 10 run ensemble mirrored that of the base forced model solution (depicted in Fig. 1) quite well, indicating that the base forced case solution is close to the center of the frequency distribution of the response and does not represent a chance outlier event.

Figures 2, 3 and 4 provide various ways of quantifying the distinct dynamical behavior of the two simulations. Figure 2 shows the observed frequency distributions of the atmospheric variable $\mathrm{X}$. In order to account for serial autocorrelations, we have selected every 30th point (the efolding decorrelation time scale of the data, representing 3.75 days) for analysis. If the two attractors were very similar then the distributions in Fig. 2 would be indistinguishable. Instead, the figure shows that the two distributions have the same mean but different shape and variance, indicating differences in the underlying attractors. These differences are

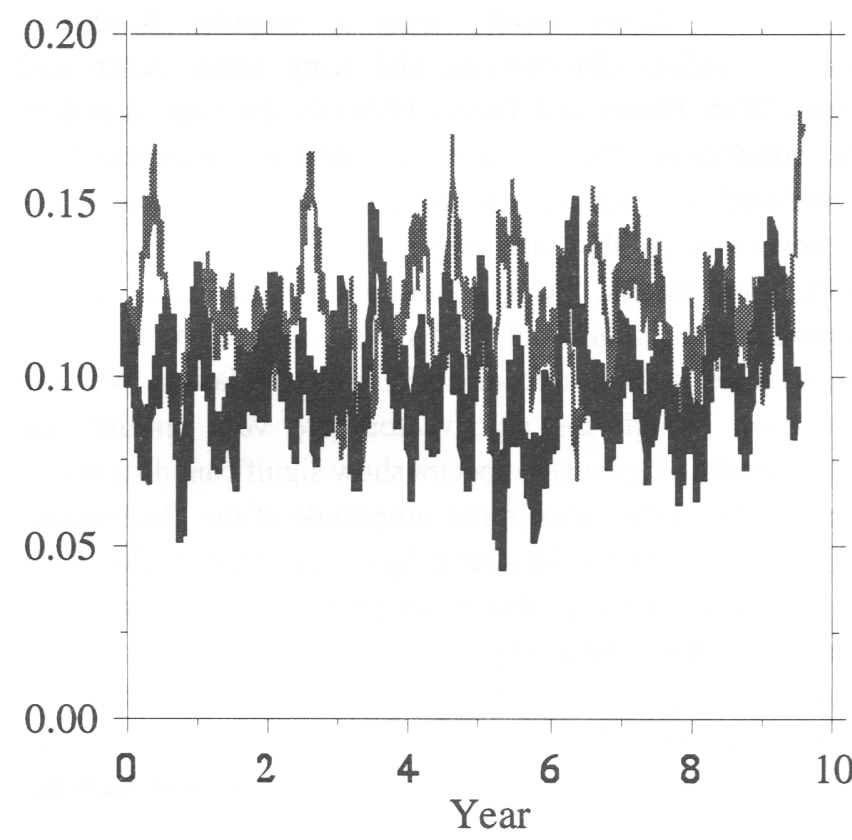

Fig. 3. The time series of the local Lyapunov exponents for the coupled (black) and forced (grey) simulations for the 10 year period.

quantified by application of the Kolmogorov-Smirnov maximum deviation test for identical populations, a standard distribution-free test for identical populations (Bradley 1968, ch. 13). The null hypothesis that the two distributions of Fig. 2 are identical is rejected at statistical significance levels above $90 \%$ (p-value is .08).

Figure 3 presents a more detailed probing of the underlying attractors by showing the local Lyapunov exponents along the two trajectories. The local Lyapunov exponents (Abarbanel et al. 1991; Brown et al. 1992) provide the local rate of divergence or convergence of nearby trajectories and therefore delineate the local intricacies of the attractor structure. Physically, they are a measure of the local predictability of the flow and the distribution of local Lyapunov exponents is thus related to the global properties of the attractor. Thus, the results of this analysis are pertinent to the structure of the variability in the two models. In both simulations, there is one positive Lyapunov exponent indicating chaotic dynamics. The average exponent is 0.20 day $^{-1}$ in the coupled simulation and 0.24 day $^{-1}$ in the forced simulation, representing a predictability of approximately 5 and 4 days, respectively. These time scales are appropriate, since we are analyzing the variability of the midlatitude general circulation (the strength of the zonal wind as represented by the variable $\mathrm{X}$ ). Locally great differences in predictability can be seen along the trajectories with low local predictability in one simulation sometimes being replaced with high local predictability in the other simulation, and vice versa. Globally, although the values of the exponents are relatively similar, the systematic offset of the two functions hints that the mean predictability is possibly not preserved in the forced simulation. 
Figure 4 shows results from a Singular Spectrum Analysis (SSA) (Broomhead and King 1986; Allen and Smith 1996; Elsner and Tsonis 1996) for the years 4 to 6 of the simulation. SSA is used to identify intermittent or modulated oscillations in time series by finding the eigenvectors (EOFs) and eigenvalues of the series logcovariance matrix. A symmetric/anti-symmetric pair of approximately sinusoidal EOFs with similar high-ranked eigenvalues is indicative of a physical oscillation. We find that even though the eigenvectors are very similar, the corresponding eigenvalue spectra show significant differences attributed to differences in the amplitude of the fluctuations in the signals. From the above figures, it appears that even though some of the global mean properties of the attractor are similar, there are always substantial local differences. As such, the variability in the forced model does not reflect the variability of the actual (coupled) system. We shall discuss the implications of these results in the next section.

\section{Discussion}

We have presented evidence that the local (space/time) variability of a fully coupled low-order atmosphere-ocean model is not the same as the local variability of the corresponding atmospheric model forced by observed SSTs. The forced system does not provide a consistent approximation to the actual local dynamics and resultant climatic variability and thus may only be reliable for the study of the global mean (attractor) behavior. Since these results reflect a fundamental aspect of the dynamical system, we expect that our conclusions will also apply to the more complex spatially extended AGCMs under current use for climate prediction. It thus becomes imperative to study the degree to which these conclusions apply to such models.

In our view, the challenge of SST dependent midlatitude extended-range forecasting is provided by the dramatically different atmospheric responses to the two quite comparable strong El Nino events in winter 1957/58 and 1972/73 (Livezey and Mo 1987). Figure 5 shows the $500 \mathrm{hPa}$ geopotential height field and deviations of those fields from the 1951-80 30 year climatology for January 1958 (Fig. 5a) and January 1973 (Fig.5b). Also shown is the $500 \mathrm{hPa}$ geopotential height difference field (1973 minus 1958, Fig. 5c). In January 1958, the midlatitude response to the warm ENSO episode followed the more classic pattern, with strong projections onto the Pacific-North American (PNA) and Tropical-Northern Hemisphere (TNH) anomaly patterns (Livezey and Mo 1987). These circulation adjustments were well reflected in observations of temperature in northwestern North America and precipitation in the southeastern United States/ Gulf of Mexico, regions identified by Ropelewski

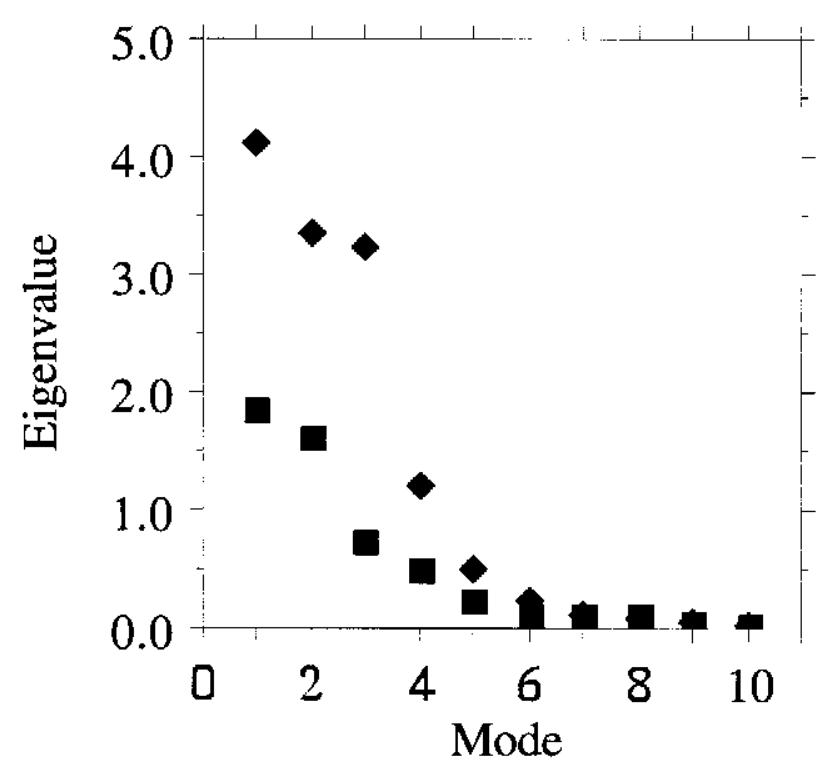

Fig. 4. Eigenvalue spectra from a Singular Spectrum Analysis (SSA) of the atmospheric model variable $\mathrm{X}$ for the coupled (O) and forced ( $\bullet$ simulations in the years 4 to 6 .

and Halpert (1986) as presenting anomalous responses to warm ENSO events (Table 1). In contrast, in January 1973, relatively weak extratropical circulation anomalies were evident and differed substantially from the classic patterns of the former episode. The enhancement of the Pacific jet in 1973 compared to 1958 is unmistakable (Fig. 5c); the jet then splits over North America (Fig. 5b), quite different than in 1958, where a much deeper long wave trough is positioned over the Great Lakes and eastern North America (Fig. 5a). These differences in the flow are not trivial and lead to very different conditions within the ENSO response regions previously noted (as indeed, across wide portions of North America). For example, Table 1 shows that temperatures in northwestern North America are much colder in 1973 than in 1958, reflecting more climatologically normal conditions for the region. Likewise, precipitation in the southeastern United States/Gulf of Mexico region was sharply reduced in 1973 compared to 1958 , and is generally more representative of climatology in that area.

Kumar and Hoerling (1995) argue that because external (SST forced) and internal variabilities are comparable at midlatitudes, ensemble averaging works to filter the "random" internal variability, leaving a clearer picture of the response to the boundary-forced signal. In this view, the considerable case-to-case variability in forecast skill for particular ENSO events is the result of the large (and unforecastable) internal variability at midlatitudes. We do not contradict this view. Rather, we argue that a potentially important factor in the boundary-forced dynamics is the character of the dynamical system itself, which we change in small but at times important ways by forcing the AGCMs 

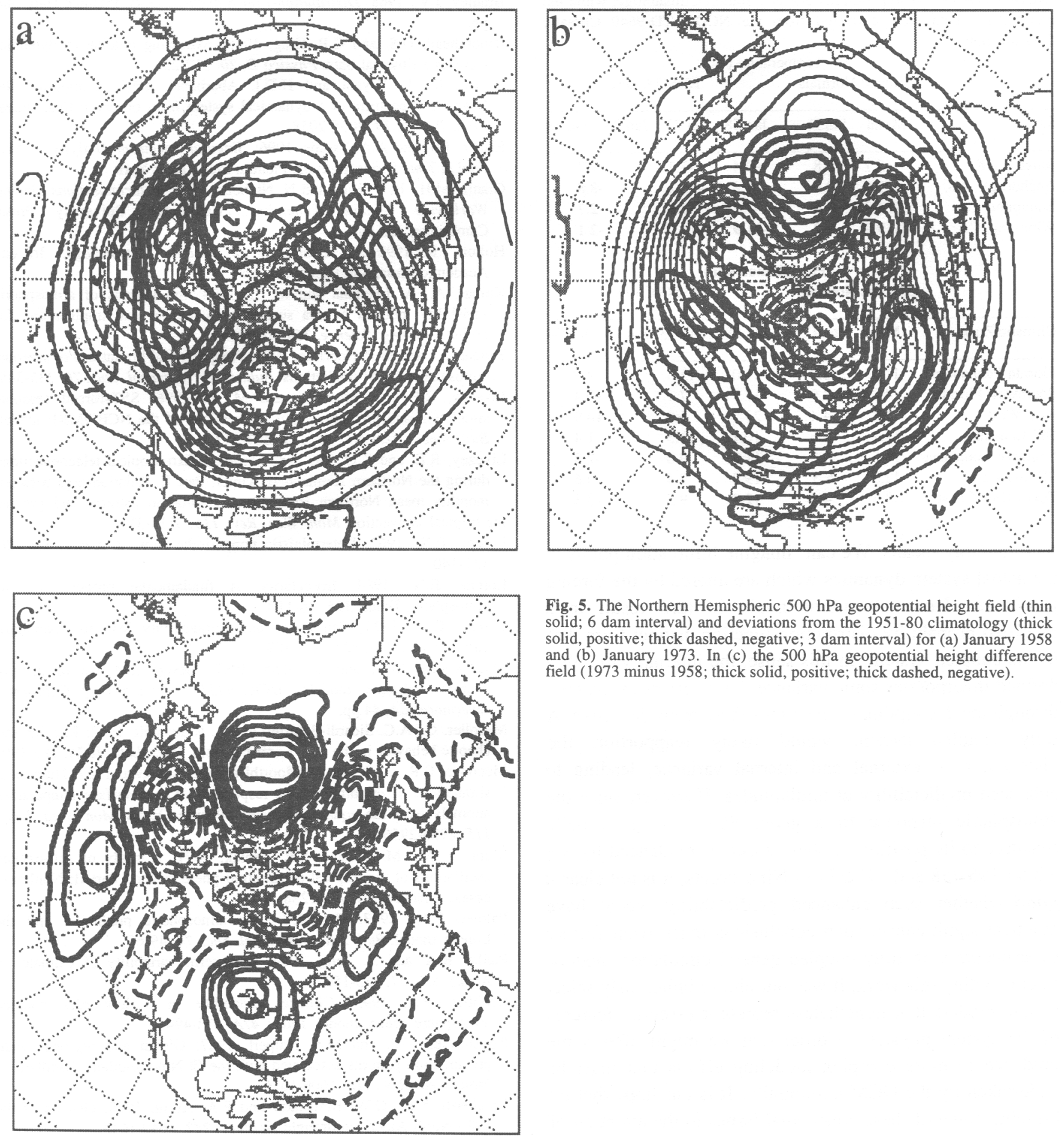

Fig. 5. The Northern Hemispheric $500 \mathrm{hPa}$ geopotential height field (thin solid; 6 dam interval) and deviations from the 1951-80 climatology (thick solid, positive; thick dashed, negative; 3 dam interval) for (a) January 1958 and (b) January 1973. In (c) the $500 \mathrm{hPa}$ geopotential height difference field (1973 minus 1958; thick solid, positive; thick dashed, negative). 
Table 1. Monthly mean observations at stations in northwestern North America (temperature) and in climatic zones in the southeastern United States (precipitiation) for two comparable warm ENSO events. Temperature data were obtained from the National Climatic Data Center (NCDC) TD-9645 World Monthly Surface Station Climatology, while the precipitation data were extracted from the NCDC TD-9640 Climatic Division Data.

Temperature $\left({ }^{\circ} \mathrm{C}\right)$
Climatic Normals

\begin{tabular}{lrrrrr}
\hline Site & Mean & Stn.D. & Jan 58 & Jan 73 & Jan 73-Jan 58 \\
\hline Juneau, AK & -5.7 & 3.8 & +0.4 & -7.3 & -7.7 \\
Anchorage, AK & -11.0 & 3.9 & -7.4 & -16.2 & -8.8 \\
Edmonton, CA & -14.9 & 4.6 & -7.2 & -9.9 & -2.7 \\
Winnipeg, CA & -19.2 & 3.2 & -11.9 & -14.0 & -2.1 \\
\multicolumn{5}{c}{ Precipitation (cm) } \\
& \multicolumn{5}{c}{ ENSO Cases }
\end{tabular}

\begin{tabular}{lrrrrr}
\hline Climatic Zone & Mean & Stn. D. & Jan 58 & Jan 73 & Jan 73 - Jan 58 \\
\hline Florida Panhandle & 11.6 & 5.7 & 11.0 & 15.3 & 4.3 \\
Southwest Florida & 4.8 & 3.9 & 17.7 & 7.3 & -10.4 \\
Key West & 4.8 & 4.4 & 21.7 & 6.8 & -14.9 \\
SW Louisiana & 12.2 & 6.6 & 10.1 & 11.5 & 1.4 \\
Southern Louisiana & 11.5 & 6.0 & 11.8 & 9.0 & -2.8 \\
SE Louisiana & 12.3 & 6.5 & 19.0 & 9.4 & -9.6 \\
Texas Gulf Coast & 8.3 & 4.5 & 12.8 & 9.5 & -3.3
\end{tabular}

with monthly means. We can imagine bifurcation points in the actual system dynamics which are altered by the forcing procedure. These alterations might in turn result in a modified probability distribution function distinct from that of the coupled system. In this sense, the internal variability defined incorporates some variance due to errors introduced through the decoupling of the atmosphere from the ocean. A fully coupled system would likely reapportion the distribution of external and internal variance, leading to enhanced predictability at midlatitudes. However, since the atmospheric response to a given SST forcing is quite sensitive to its position in phase space as evidenced by the winter $1957 / 58$ and 1972/73 El Nino events, it is not clear $a$ prion whether such enhanced predictability would have practical significance; such conclusions must await further experiments with fully coupled general circulation models. In conducting this work, it was our aim to judiciously select simple models that nonetheless represent essential features of the system physics, in order that we might offer some guidance to more complex modeling efforts that must be undertaken by the GCM community. It is our hope that by bringing some of these conceptual issues to the attention of that community that we will help stimulate higher-order modeling efforts.

Acknowledgments. This work was partially supported by the National Science Foundation under ATM-9310959. We wish to thank E. Tziperman for his help in implementing the heuristic ENSO model.

\section{References}

exponents in chaotic systems: Their importance and their evaluation using observed data. Int. J. Mod. Phys., 5, 1347-1375.

Allen, M.R. and L.A. Smith, 1996: Monte Carlo SSA: Detecting irregular ascillations in the presence of coloured noise. $J$. Climate, in press.

Bradley, J.V., 1968: Distribution-Free Statistical Tests. Prentice Hall, 388 pp.

Broomhead, D.S. and G.P. King, 1986: Extracting qualitative dynamics from experimental data. Physica $D, 20,217-236$.

Brown, R., P. Bryant and H.D.I. Abarbanel, 1992: Computing the Lyapunov spectrum of a dynamical system from observed time series. Phys. Rev. A, 19, 2055-2058.

Elsner, J.B. and A.A. Tsonis, 1996: Singular Spectrum Analysis: A New Tool in Time Series Analysis. Plenum Press, New York.

Glantz, M.H., R.W. Katz and N. Nicholls, 1991: Teleconnections Linking Worldwide Climate Anomalies. Cambridge University Press, Cambridge, 353 pp.

Holton, J.R., 1992: An Introduction to Dynamic Meteorology, 3rd ed., Academic Press, San Diego, 511 pp.

Kumar, A., A. Leetmaa and M. Ji, 1994: Simulations of atmospheric variability induced by sea surface temperatures and implications for global warming. Science, 266, 632-634.

Kumar, A., and M.P. Hoerling, 1995: Prospects and limitations of seasonal atmospheric GCM predictions. Bull. Amer. Meteorol. Soc., 76, 335-345.

Livezey, R.E., M. Masutani and M. Ji, 1996: SST-forced seasonal simulation and prediction skill for versions of the NCEP/MRF model. Bull. Amer. Meteorol. Soc., 77, 507-517.

Livezey, R.E. and K.C. Mo 1987: Tropical-Extratropical teleconnections during the Northern Hemisphere winter. Part II: Relationships between monthly mean Northern Hemisphere circulation patterns and proxies for tropical convection. Mon. Wea. Rev., 115, 3115-3132.

Lorenz, E.N., 1963: Deterministic nonperiodic flow. J. Atmos. Sci, 20, $131-140$.

Lorenz, E.N., 1984: Irregularity. A fundamental property of the atmosphere. Tellus, 36A, 98-110.

Lorenz, E.N., 1990; Can chaos and intransitivity lead to interannual variability? Tellus, 42A, 378-389.

MacCracken, M.C. and S. Ghan, 1987: Design and use of zonally averaged climate models. UCRL-94338, University of California, Livermore, CA, $44 \mathrm{pp}$.

Masoller, C., A.C. Sicardi-Schifino and L. Romanelli, 1992: Regular and chaotic behavior in the new Lorenz system. Phys. Lett., 167A, 185-190.

Mechoso, C.R., A. Kitoh, S. Moorthi and A. Arakawa, 1987: Numerical simulations of the atmospheric response to a seas surface temperature anomaly over the equatorial eastern Pacific Ocean. Mon. Wea. Rev., $115,2936-2956$.

Munnich, M., M.A. Cane, S.E. Zebiak, 1991: A study of self-excited oscillations of the tropical ocean-atmosphere system. Part II: Nonlinear cases. J. Atmos. Sci., 48, 1238-1248.

Palmer, T.N., 1993: Extended-range atmospheric prediction and the Lorenz model. Bull. Amer. Meteorol. Soc., 74, 49-65.

Pielke, R.A. and X. Zeng, 1994: Long-term variability of Climate. $J$. Atmos. Sci., 51, 155-159.

Roebber, P.J., 1995: Climate variability in a low-order coupled atmosphere-ocean model. Tellus, 47A, 473-494.

Ropelewski, C.F. and M.S. Halpert, 1987: Global and regional scale precipitation patterns associated with the $\mathrm{El} \mathrm{Nino/Southem} \mathrm{Oscillation.}$ Mon. Wea. Rev., 115, 1606-1626.

Trenberth, K.E., G.W. Branstator and P.A. Arkin, 1988: Origins of the 1988 North American drought. Science, 242, 1640-1645.

Tziperman, E., L. Stone, M.A. Cane and H. Jarosh, 1994: El Nino chaos: overlapping of resonances between the seasonal cycle and the Pacific Ocean-atmosphere oscillator. Science, 264, 72-74.

Weaver, A.J. and T.M.C. Hughes, 1992: Stability and variability of the thermohaline circulation and its link to climate. Trends in Physical Oceanography, Council of Scientific Research Integration, Research Trends Series, Trivandrum, India.

Zebiak, S.E. and M.A. Cane, 1987: A model El Nino-Southern Oscillation. Mon. Wea. Rev., 115, 2262-2278. 